\title{
Efficacy of Intraventricular Sponge Placement to Capture Debris in Aortic Valve Replacement
}

\author{
Kyle Purrman ${ }^{1}$, Hossein Amirjamshidi ${ }^{1}$, Chauncey Syposs ${ }^{1}$, Courtney Vidovich ${ }^{1}$, Brendan \\ Boyce $^{1}$, and Peter Knight ${ }^{2}$ \\ ${ }^{1}$ University of Rochester Medical Center \\ ${ }^{2}$ University of Rochester
}

February 12, 2021

\begin{abstract}
Background: Surgical aortic valve replacement (SAVR) carries the known risk of shedding debris into the left ventricle during valve leaflet excision and annulus debridement. Embolization of this debris may have devastating effects for the patient. While surgeons have developed methods to mitigate this risk, no data exists as to their efficacy. Herein, we present the first study that evaluates the efficacy of a technique for capturing debris during SAVR. Methods: Our group conducted a prospective case series of 20 patients who underwent SAVR using the insertion of an intraventricular surgical sponge prior to valve leaflet excision and annulus debridement to capture debris. Surgical sponges were grossly, radiographically, and histologically examined for the presence of cellular and acellular debris to determine the efficacy of this technique. Results: Of the 20 surgical sponges analyzed, 15 (75\%) specimens registered positivity for cellular and/or acellular debris. 7 (35\%) sponges were grossly positive, 15 (75\%) were radiographically positive, and $4(20 \%)$ were histologically positive for calcified debris on examination. Conclusions: This represents the first study that objectively evaluates a method used to capture debris in SAVR procedures. Our results demonstrate a high frequency of debris captured within intraventricular surgical sponges and confirms the efficacy of this technique. While this data is promising, numerous additional approaches exist to capture debris and a best practice standard should exist across the specialty. In addition, this study does not address the clinical outcomes associated with this technique. To these ends, additional data and multicenter collaboration is required.
\end{abstract}

TITLE: Efficacy of Intraventricular Sponge Placement to Capture Debris in Aortic Valve Replacement RUNNING HEAD: Efficacy of Surgical Sponge in Capturing Debris

Kyle C Purrman MD ${ }^{1}$, Hossein Amirjamshidi MD ${ }^{1}$, Chauncey Syposs $\mathrm{DO}^{2}$, Courtney Vidovich BS ${ }^{1}$, Brendan Boyce $\mathrm{MD}^{2}$, Peter Knight $\mathrm{MD}^{1}$

Division of Cardiac Surgery ${ }^{1}$, Division of Pathology ${ }^{2}$, University of Rochester Medical Center, Rochester NY 14620

Data Availability Statement: Deidentified data may be made available from the corresponding author, upon reasonable request.

Funding: None

Conflict of Interest: None to disclose

IRB Approval: STUDY00004551, Approved 3/30/2020

CORRESPONDING AUTHOR:

Kyle C Purrman, MD 
Cardiothoracic Surgery Residency

University of Rochester Medical Center

601 Elmwood Ave., Box SURG

Email: kyle_purrman@urmc.rochester.edu

\section{ABSTRACT:}

\section{Background :}

Surgical aortic valve replacement (SAVR) carries the known risk of shedding debris into the left ventricle during valve leaflet excision and annulus debridement. Embolization of this debris may have devastating effects for the patient. While surgeons have developed methods to mitigate this risk, no data exists as to their efficacy. Herein, we present the first study that evaluates the efficacy of a technique for capturing debris during SAVR.

\section{Methods :}

Our group conducted a prospective case series of 20 patients who underwent SAVR using the insertion of an intraventricular surgical sponge prior to valve leaflet excision and annulus debridement to capture debris. Surgical sponges were grossly, radiographically, and histologically examined for the presence of cellular and acellular debris to determine the efficacy of this technique.

\section{Results:}

Of the 20 surgical sponges analyzed, 15 (75\%) specimens registered positivity for cellular and/or acellular debris. $7(35 \%)$ sponges were grossly positive, $15(75 \%)$ were radiographically positive, and $4(20 \%)$ were histologically positive for calcified debris on examination.

\section{Conclusions:}

This represents the first study that objectively evaluates a method used to capture debris in SAVR procedures. Our results demonstrate a high frequency of debris captured within intraventricular surgical sponges and confirms the efficacy of this technique. While this data is promising, numerous additional approaches exist to capture debris and a best practice standard should exist across the specialty. In addition, this study does not address the clinical outcomes associated with this technique. To these ends, additional data and multicenter collaboration is required.

\section{MANUSCRIPT:}

\section{Introduction:}

Surgical aortic valve replacement (SAVR) has been the gold standard treatment for severe aortic stenosis for the past several decades and remains one of the most commonly performed operations for the practicing adult cardiac surgeon. ${ }^{1}$ Despite SAVR being a relatively standard operation, it is not without significant risk to the patient. This risk is attributed to, in part, by the innate invasiveness of the procedure and the comorbidities common within the patient population. SAVR also carries a well-known risk of shedding debris into the left ventricle during aortic valve leaflet excision and annulus debridement. Embolization of this debris into systemic circulation may cause a wide range of complications including ischemic complications of the brain, visceral organs, and extremities. ${ }^{2}$ Recent meta-analyses concerning early postoperative complications for SAVR indicate that $58 \%$ of patients experience a silent brain infarct and $5.1 \%$ of patients experience a TIA or stroke as a sequelae of this procedure. ${ }^{2-3}$ These complications may have devastating effects on the lives of patients, and the surgeon must take it upon themself to mitigate these risks to patients.

Numerous techniques have been developed to capture debris developed during aortic valve leaflet removal and annulus debridement. Most commonly, delicate surgical debridement, left ventricular irrigation with suctioning, and insertion of intraventricular surgical sponges have been employed for this purpose. ${ }^{4}$ Several 
additional techniques exist that are often practice specific and the superiority of specific approaches is a contentious and hotly debated topic. Though numerous techniques exist, no objective data currently exists in the literature to address their relative efficacy. The value of these techniques has recently been called into question. For example, our group recently published an ex-vivo porcine model evaluating the efficacy of left ventricle irrigation and careful suctioning to capture debris. Debris of varying density (limestone pieces, pledgets, and tissue) were placed inside the left ventricle (LV) and the LV was thoroughly irrigated and suctioned numerous times. The resulting solutions were filtered and the debris within them was quantified. It was found that the rate of debris capture was relatively low, $43 \%$ of pledgets, $17 \%$ of limestone, and $9 \%$ of tissue pieces were retrieved. ${ }^{4}$ Given the devastating nature of these complications, the individual and relative efficacy of each technique aimed at capturing calcified debris during SAVR should be objectively evaluated, compared, and a best practice standard should be developed for use across the specialty.

The insertion of a surgical sponge into the left ventricle prior to aortic valve leaflet excision and annulus debridement is a simple and cost-effective method for capturing debris developed during SAVR (Figure 1). ${ }^{5}$ Herein, we present a prospective case series aimed at objectively evaluating the efficacy of this technique in capturing debris via gross, radiographic, and histological examination of surgical specimens.

\section{Methods:}

This study is a single-center, prospective case series of 20 patients who underwent surgical aortic valve replacement (SAVR) between January 2020 - July 2020 at the University of Rochester Medical Center (URMC). International Review Board (IRB) approval was obtained prior to commencing data collection. A waiver of informed consent was approved by the reviewing IRB. All patients who underwent elective surgical aortic valve replacement for severe aortic stenosis (mean gradient $>40 \mathrm{mmHg}$, AVA $<1 \mathrm{~cm}^{2}, \operatorname{Vmax}>4$ $\mathrm{m} / \mathrm{s}$ ) using the below technique were included. Pediatric patients $(<18$ years old) and those with mixed aortic valve pathology were excluded from this study.

The technique used for capturing debris developed during aortic valve leaflet removal and annulus debridement in SAVR for each of the 20 patients in our prospective case series is described below (Figure 1). Following the establishment of cardiopulmonary bypass, diastolic arrest of the heart and aortotomy, a surgical sponge is inserted into the left ventricle through the aortic valve. The aortic valve leaflets are then carefully excised, and the aortic annulus is meticulously debrided in the usual fashion. The left ventricle is then copiously irrigated with normal saline which is suctioned from the left ventricular cavity. The surgical sponge is then removed from the heart and sent to Surgical Pathology for gross, radiographic, and histologic analysis (as below). It is important to note that closed loop communication is used between the surgeon and operating room staff to confirm both the placement and removal of the surgical sponge during the procedure. The remainder of the procedure then proceeds in the usual fashion.

In the surgical pathology lab, the surgical sponges were grossly examined for any signs of debris, and then were radiographically imaged using a Kubtec XPERT radiography system. Unused sponges were also imaged as negative controls. Samples were then vigorously washed with isotonic saline in order to liberate debris and were then processed into cell blocks using standard protocols. The tissue blocks were stained with Hematoxylin and Eosin and von Kassa Calcium Stain (Figure 2). The resulting slides were then reviewed by two different Pathologists and scored quantitatively as positive or negative for the presence of acellular and cellular tissue and calcified debris. Positive controls for von Kassa were analyzed and deemed to be adequate. Quantitative information and photography were generated with Olympus BX45 microscopes using CellSens imaging software.

\section{Results:}

The demographic characteristics of our patient population are summarized in Table 1. The surgical sponges used in each of the SAVR procedures to capture debris were analyzed in the surgical pathology lab as described above. The results of the examination of the surgical specimens are summarized in Table 2. Of note, $15(75 \%)$ of surgical sponges registered positivity for some degree of cellular and/or acellular debris. $7(35 \%)$ of sponges were positive for calcified debris on gross examination of the specimen. On 
radiographic examination using a Kubtext XPERT radiography system, 15 (75\%) were shown to be positive for debris. Histologic examination using Hematoxylin and Eosin and von Kassa Calcium stain revealed that $5(25 \%)$ of specimens registered histologic positivity for calcified debris, indicating the presence of calcified atherosclerotic plaque. Given the discrepancy between radiographic positivity and histologic positivity, our finding of $25 \%$ of samples having calcified debris is likely an underestimate. Representative images from the pathologic analysis of surgical specimens are shown in Figure 2.

\section{Comment:}

The results of our study demonstrate a high frequency of debris captured within surgical sponges placed in the left ventricle prior to aortic valve leaflet removal and annulus debridement. $75 \%$ of the sponges used in these procedures had some form of cellular or acellular debris contained within them on examination. 35\% of sponges were grossly positive, $75 \%$ were radiographically positive, and $25 \%$ were histologically positive for calcified debris. The discrepancy between radiographic and histologic detection of calcified debris likely results from loss of the specimen during transfer or from under sampling the blocks from which microscopic slides are made. Alternatively, specimens may also contain surgical debris that is not mineralized. The results of this study are certainly encouraging with respect to this specific technique. However, this study was not designed to understand the possible clinical implications of embolization of the captured debris into systemic circulation. It is unclear if, for example, embolization of debris that is only able to be detected radiographically or histologically would have resulted in clinically significant complications for the patient. More data is needed to fully appreciate this relationship. Additionally, because calcified debris does not maintain its shape when cut for microscopic sections, our current methodological design cannot reliably estimate embolus size, though observed calcified debris ranged from $0.1 \mathrm{~mm}$ through $0.33 \mathrm{~mm}$ (Figure 3). However, we believe that consensus among practicing Cardiac Surgeons would support decreasing calcified debris of any size as a beneficial outcome for the patient.

This is the first published study aimed at objectively evaluating the efficacy of a technique for decreasing intravascular calcified debris in surgical aortic valve replacement. While the data in this proof-of-concept study is promising with respect to this technique, numerous additional techniques exist across practices and centers aimed at the same purpose. Given the devastating nature of these complications, the individual and relative efficacy of the prevention techniques should be objectively evaluated, compared, and a best practice standard should be developed for use across the specialty. To this end, additional data and multi-center collaboration is required.

\section{References}

1. Chien, S., Clark, C., Maheshwari, S., Koutsogiannidis, C. P., Zamvar, V., Giordano, V., \& Pessotto, R. (2020). Benefits of rapid deployment aortic valve replacement with a mini upper sternotomy. Journal of Cardiothoracic Surgery, 15 (1), 1-6.

2. Indja B, Woldendorp K, Vallely MP, Grieve SM. Silent Brain Infarcts Following Cardiac Procedures: A Systematic Review and Meta-Analysis.J Am Heart Assoc . 2019;8(9):e010920. doi:10.1161/JAHA.118.010920

3. Indraratna P, Tian DH, Yan TD, Doyle MP, Cao C. Transcatheter aortic valve implantation versus surgical aortic valve replacement: A meta-analysis of randomized controlled trials. Int $J$ Cardiol . 2016;224:382-387. doi:10.1016/j.ijcard.2016.09.018

4. Amirjamshidi H, Sauer J, Hand C, Knight P. Is there a role for irrigation of the left ventricle during aortic valve surgery? ISMICS 2020 Abstract.

5. Ehsan, A., \& Sellke, F. W. (2019). Chapter 9 - Aortic Valve Replacement. In F. W. Sellke \& M. Ruel (Eds.), Atlas of Cardiac Surgical Techniques (Second Edition) (pp. 129-139): Elsevier. 

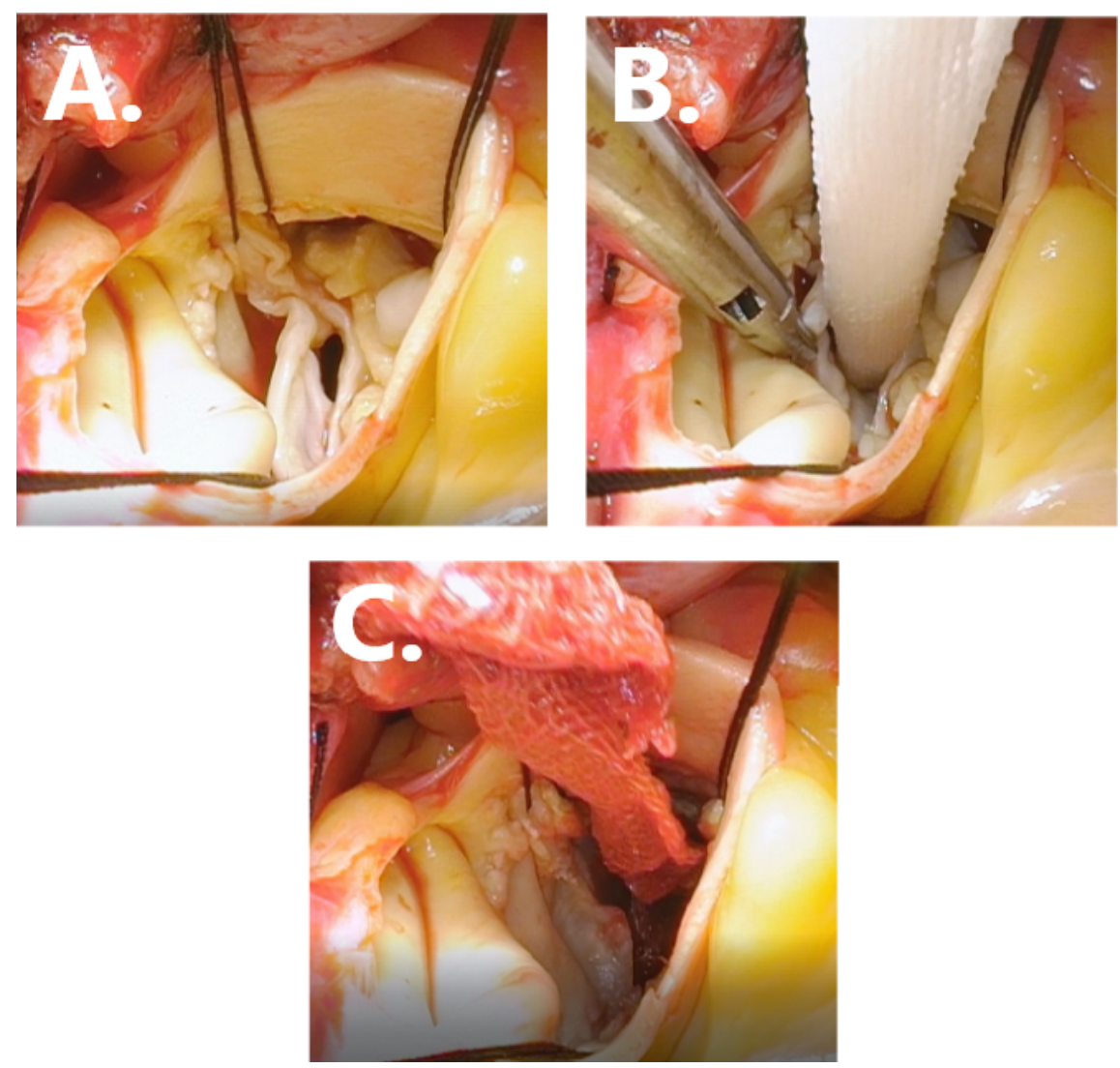


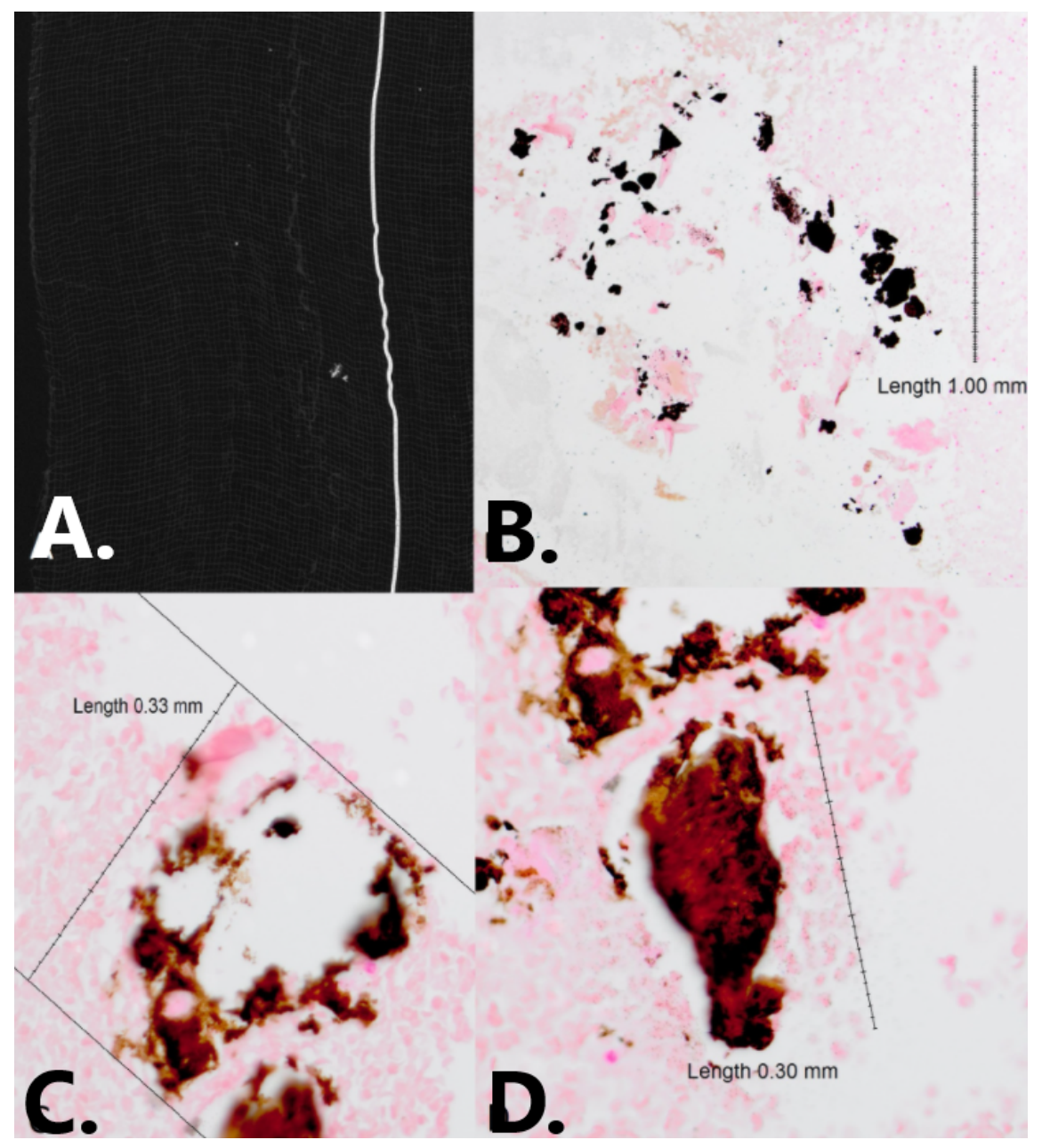


Table 1. Characteristics of 20 patients undergoing AVR

\begin{tabular}{lc}
\hline Variable & $\begin{array}{c}\text { Value } \\
\mathrm{n}(\%) \text { or Mean } \pm \mathrm{SD}\end{array}$ \\
\hline Age, $\mathrm{y}$ & $62.8 \pm 9.1$ \\
Male, $\mathrm{n}(\%)$ & $13(65)$ \\
Comorbidities & \\
Hypertension & $16(80)$ \\
PAD & $2(10)$ \\
CAD & $9(45)$ \\
Prior MI & $3(15)$ \\
Arrhythmia & $7(35)$ \\
Diabetes & $8(40)$ \\
Dyslipidemia & $13(65)$ \\
History of smoking & $12(60)$ \\
Obesity (BMI $\left.>30 \mathrm{~kg} / \mathrm{m}^{2}\right)$ & $12(60)$ \\
Heart Failure & $3(15)$ \\
Stroke & $2(10)$ \\
Renal dialysis & $1(5)$ \\
COPD & $0(0)$ \\
Baseline Echocardiogram data & \\
Aortic valve area, cm & \\
Peak gradient, mmHg & $0.752 \pm 0.195$ \\
Mean gradient, mmHg & $73.6 \pm 19.6$ \\
EOAI & $41.6 \pm 15.3$ \\
Aortic insufficiency & $0.367 \pm 0.093$ \\
Mitral regurgitation & $14(70)$ \\
Left ventricle ejection fraction, \% & $17(85)$ \\
Procedure & $60.7 \pm 13.3$ \\
STS mortality risk score, \% & \\
Right anterior thoracotomy & $1.72 \pm 1.43$ \\
Sternotomy & $18(90)$ \\
SD, standard deviation; CAD, coronary artery disease; EOAI, effective orifice area index; MI, \\
myocardial infarction; PAD, peripheral arterial disease; STS, Society of Thoracic Surgeons \\
\end{tabular}


Analysis Method

Cellular/Acellular Debris

Grossly visible

Radiographic

Histologic
No. of Cases Positive ( $\%)$

$15(75 \%)$

$7(35 \%)$

$15(75 \%)$

$5(25 \%)$ 